\title{
VARIATION IN THE MINERAL COMPOSITION OF RYE SEEDLINGS
}

\author{
H. REES, M. A. BALUCH and D. B. JAMES \\ Department of Agricultural Botony, University College \\ of Woles, Aberystwyth
}

Received 30.xi.60

\section{INTRODUCTION}

ThE improvement of crop quality by the plant breeder is achieved directly or indirectly by selection for heritable chemical differences in the composition of individual phenotypes. Improvement of yield must also inevitably result in the selection of chemical differences either as causes or consequences of vigorous plant development. This being so it is perhaps surprising that comparatively little attention has been devoted, in higher plants at any rate, to investigating directly the variation in chemical composition of plants within and between populations (see Wagner and Mitchell, r955).

The present work was the beginning of an inquiry into the variation in plant mineral composition occurring within a population. For this purpose a comparison was made between three rye families each derived by inbreeding from the same initial population-the variety Stålråg. The advantage of using inbred families is that potential variation is released when inbreeding is imposed on normally outbreeding populations such as occur in rye. This greatly facilitates the detection of the inherent variability of the original population. In this work the minerals investigated were potassium, phosphorus and iron.

\section{MATERIAL AND METHOD}

As mentioned earlier the three families used were all derived from the Swedish variety Stålråg. Two were homozygous lines, $P 6$ and $P_{I 7}$, inbred by self-pollination for more than thirty generations. The third family was an $F_{2}$ produced by selfpollinating an $F_{I}$ hybrid between $P 6$ and another line.

Seedlings were raised in soil (J.I. Potting Compost) in boxes. Five weeks after sowing the shoots of each plant were cut off complete and analysed separately for the three mineral components. At this stage all plants were at a corresponding stage of development, each having developed three vegetative tillers. The methods for mineral analysis were as follows :-

I. Plants were dry-ashed in a muffle furnace, according to the method of Peech (see Jackson, 1958).

2. The plant extract was taken up in 2 per cent. hydrochloric acid.

3. Potassium was estimated on an Eel flame photometer.

4. Phosphorus determination was by the stannous chloride-reduced molybdenum blue method (Jackson, 1958).

5. Iron was estimated using Bathophenanthroline (Smith, McGurdy and Diehl, 1952). 


\section{RESULTS}

The means and variances of dry weights of shoots and of the quantities of the three minerals are given in table $I$. The data are also presented in the graphs in figs. I, 2 and 3.

In analysing and interpreting these results it is necessary to pay special attention to two aspects of variation that are immediately apparent from the table and graphs. First, it will be seen that dry weights vary between families. Second, the total mineral content, as we should expect, increases with weight. It follows, therefore, that while obvious family differences in mineral composition are revealed by the seedlings these differences could, on the face of it, reflect merely

TABLE I

Means ( $(\bar{\alpha})$, Variances $(V)$ and co-variances $(W \mathrm{xy})$ of mineral contents $(\mathrm{y})$ and dry weights $(\mathrm{x})$ of seedlings in three rye families. Numbers of plants analysed are given in brackets

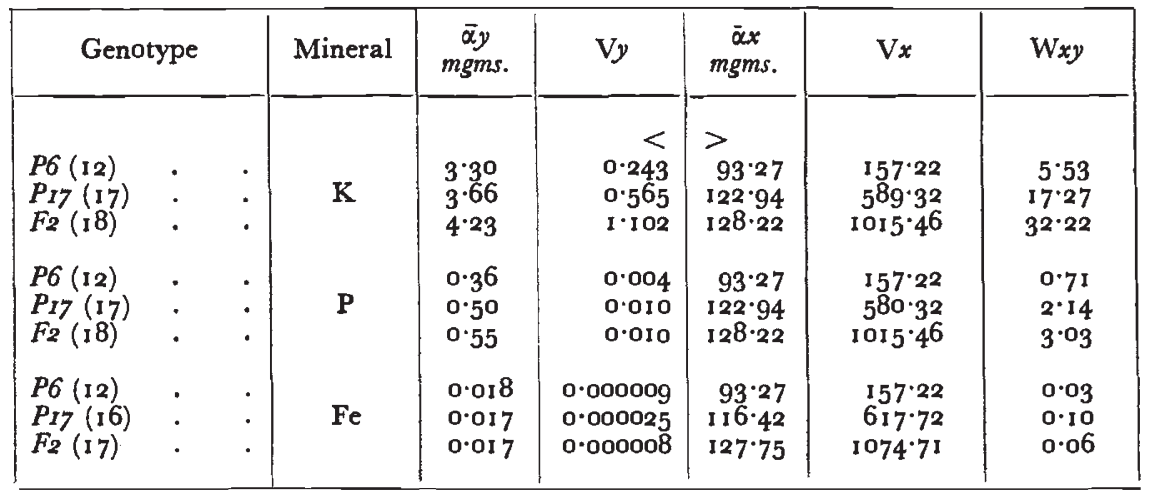

Although co-variances $(W x y)$ are given for each mineral the relation between phosphorus and weight is in fact curvilinear, not linear (see text).

a variation in growth rate between the genotypes. What we need to know, however, and what must determine the statistical approach to analysing the data is whether genotypes vary in mineral composition independently of their growth rates. The method and the results of the analysis appear below.

(a) Potassium. The average potassium values for families, which are given in the table, differ significantly from one another, but, as mentioned above, these values are also correlated with the variation in average weight of families. The correlation is illustrated clearly in fig. I, which shows the general increase in potassium associated with increasing weight.

To determine, now, whether family differences in potassium content exist independently of weight we note two features of the graph that are of special significance. First, the relation between the two characters within each family is linear-the joint regression being significant at the $0 \cdot I$ per cent. level. Second, an analysis of variance shows no significant heterogeneity between the regressions in $P 6, P_{I 7}$ and 
the $F 2$. The $b$ values are, respectively, $0.035,0.030$ and 0.032 . These two facts establish that the rate of change of potassium content on weight is consistent within families over the range of seedling weights represented. Consequently, by removing that part of the variation dependent on weight, which is common to all families, we can compare the families in respect of the residual variation in potassium that is

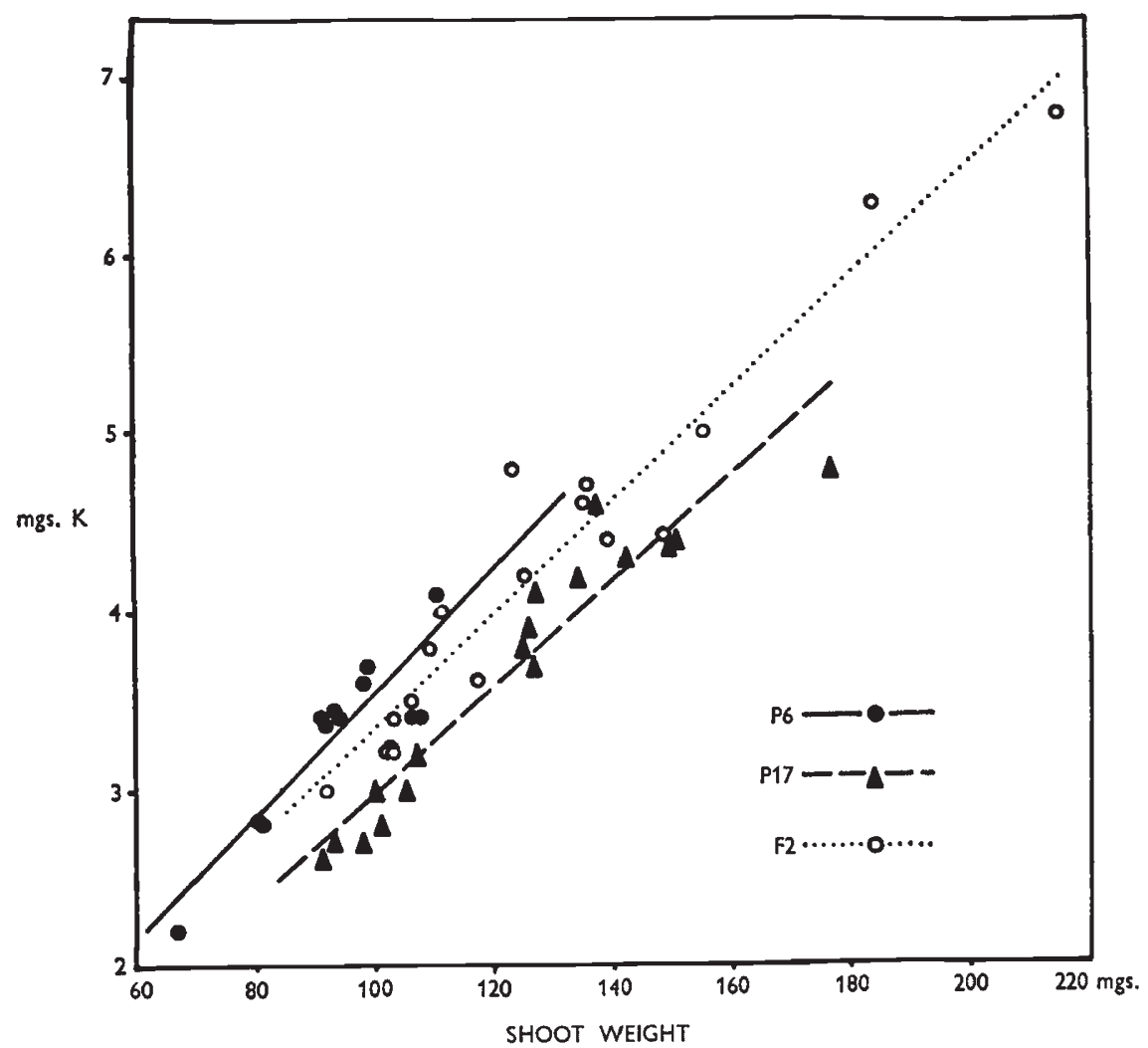

Fig. 1.-Potassium content of shoots of rye seedlings plotted against weight.

independent of seedling weight. A co-variance analysis serves the purpose admirably.

The analysis is set out in table 2. As will be seen the residual variation between genotypes is highly significant $(P=<0.001)$. For corresponding weights, as the graph shows, $P 6$ has the most, $P_{r 7}$ the least, potassium in its seedlings.

(b) Phosphorus. The graph in fig. 2 indicates a pattern of variation for phosphorus which in many respects is similar to that described for potassium. There is an increase in amount with increasing weight and there appear to be differences in phosphorus quantities for plants with corresponding weights from different families. In one respect, however, the phosphorus graph differs from the potassium one. While 
the latter shows a linear increase in mineral quantity with increasing weight, that for phosphorus indicates a curvilinear relation with weight. A polynomial regression analysis of the data confirms an

TABLE 2

The co-variance analysis of potassium content $(\mathrm{y})$ and seedling weight $(\mathrm{x})$

\begin{tabular}{|c|c|c|c|c|c|c|c|c|c|}
\hline & $\mathrm{SS} x$ & $\mathrm{~S}_{x y}$ & $\mathrm{SS} y$ & SS regr. & SS res. & & & & \\
\hline Total & $37,893 \cdot 31$ & I $100 \cdot 15$ & $37 \cdot 11$ & $31 \cdot 94$ & $5 \cdot 17$ & $\mathbf{N}$ & MS & VR & $P$ \\
\hline Genotypes & 9,6 I $5 \cdot 88$ & 215.23 & $6 \cdot 66$ & $\cdots$ & $2 \cdot 41$ & 2 & $I \cdot 2 I$ & $20 \cdot 17$ & 0.001 \\
\hline Error . & $28,277 \cdot 43$ & $884 \cdot 92$ & $30 \cdot 45$ & $27 \cdot 69$ & $2 \cdot 76$ & 43 & 0.06 & $\cdots$ & $\cdots$ \\
\hline
\end{tabular}

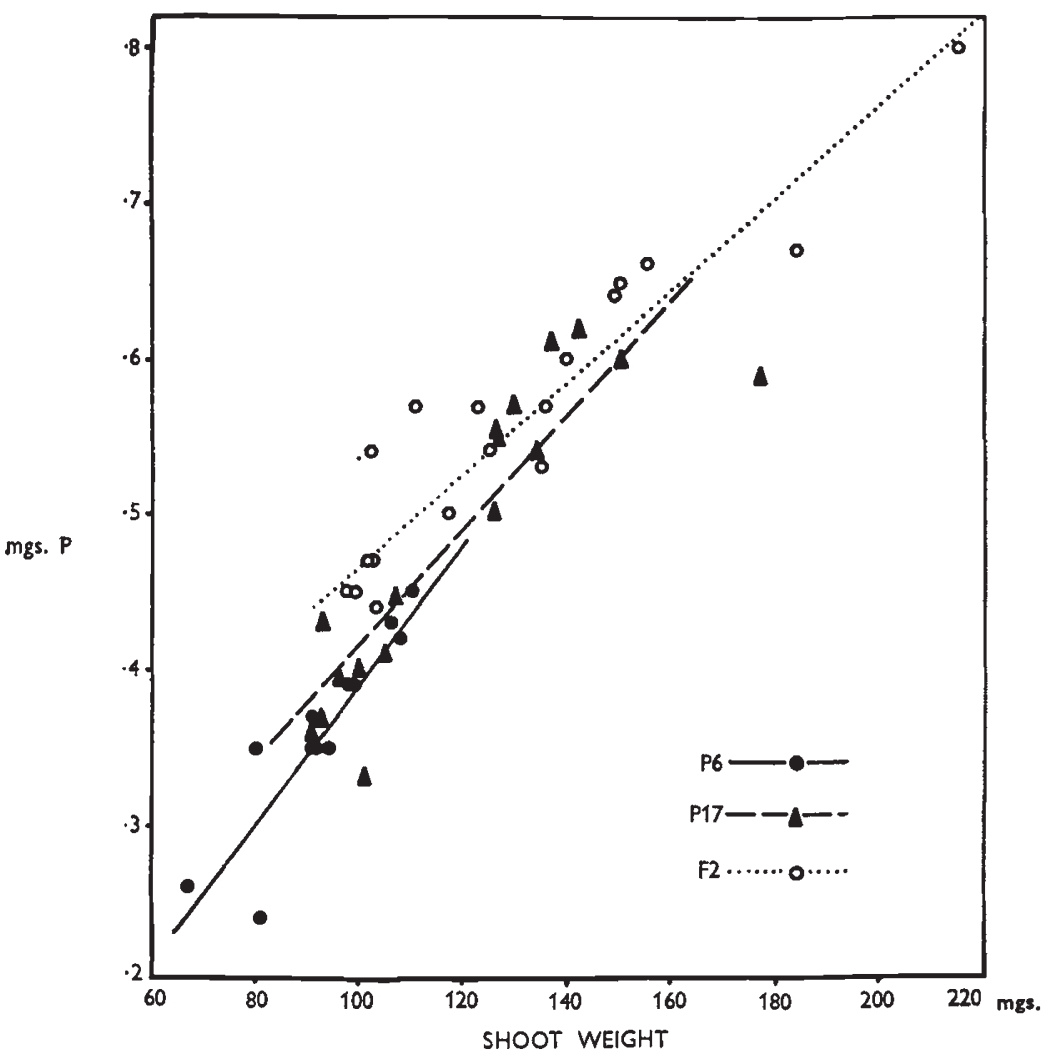

Fig. 2.-Phosphorus content of shoots of rye seedlings plotted against weight. For convenience the linear regressions have been plotted, whereas the relation between mineral and weight is in fact curvilinear (see text).

overall curvilinearity $(P=<0 \cdot 01)$. In view of this it was clearly necessary to take curvilinearity into account when analysing, by co-variance, the phosphorus data. In the analysis, table 3 , the 
regression sums of squares consequently include both linear and quadratic items.

It will be seen from the analysis that phosphorus content, over and

TABLE 3

The co-variance analysis of phosphorus content $(\mathrm{y})$ on seedling weight $(\mathbf{x})$. The regression sums of squares include both linear and quadratic components (see text)

\begin{tabular}{|c|c|c|c|c|c|c|c|c|}
\hline & $\mathrm{SS} y$ & SS regr. & SS res. & & & & & \\
\hline Total. & 0.6427 & 0.5632 & 0.0795 & $\mathrm{~N}$ & MS & VR & $\mathbf{P}$ & \\
\hline Genotypes & ... & ... & $0.014^{8}$ & 2 & 0.0074 & $4 \cdot 93$ & 0.02 & -0.01 \\
\hline Error & $0 \cdot 3888$ & $0.324 \mathrm{I}$ & 0.0647 & 42 & 0.0015 & $\ldots$ & $\ldots$ & $\ldots$ \\
\hline
\end{tabular}

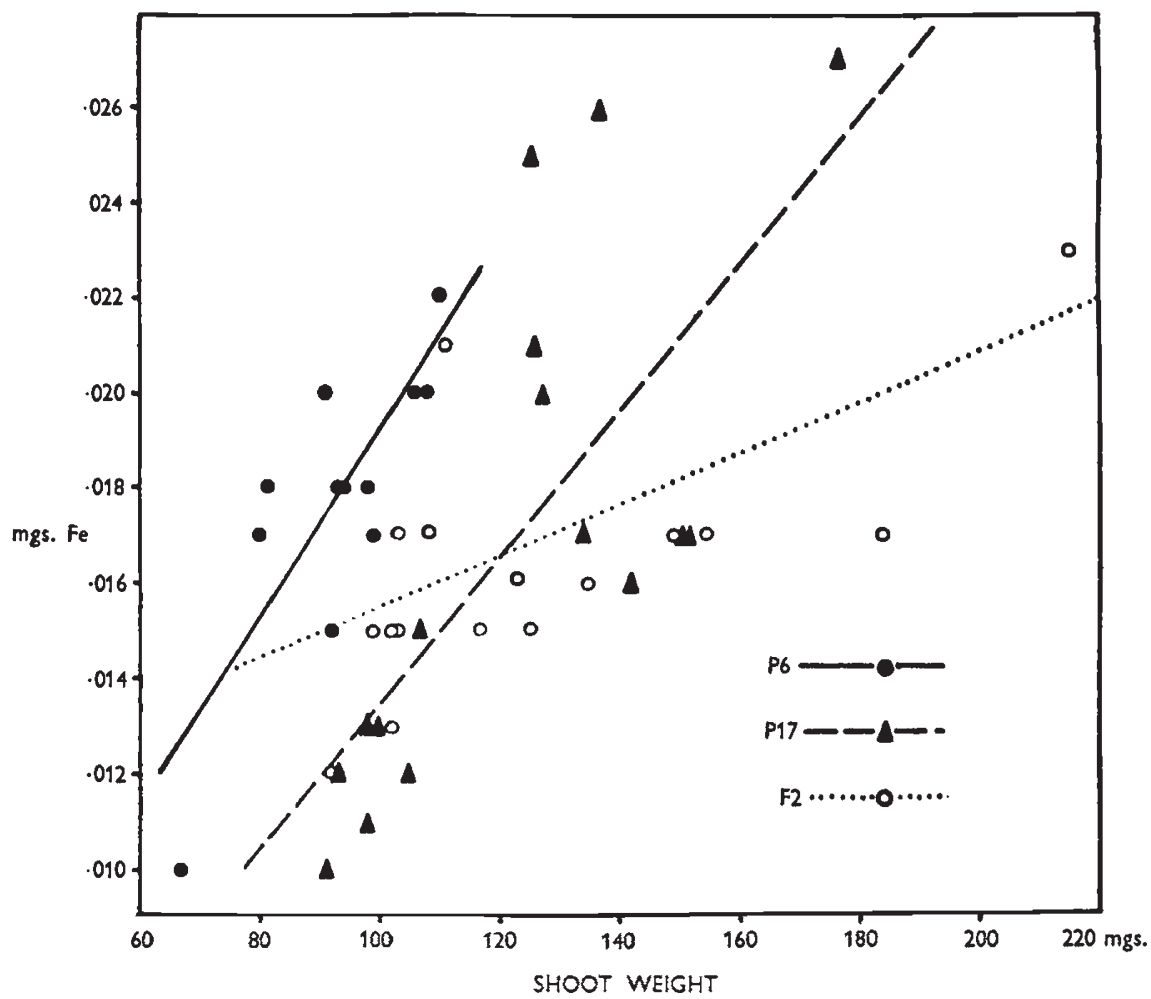

FIG. 3.-Iron content of shoots of rye seedlings plotted against weight.

above that accounted for by regression is significantly different between families.* For corresponding weights (fig. 2) $F_{2}$ seedlings contain the most phosphorus, $P 6$ seedlings the least.

* It is worth emphasising that in assessing and comparing the mineral compositions of plants the use of percentages, as is often adopted, to express content can be grossly misleading. This is especially true where content varies in curvilinear relation with weight. In such cases, differences in percentage values may reflect merely differences correlated with varying rates of development. 
(c) Iron. The situation for iron (see fig. 3) is rather less straightforward than for either of the two minerals above. There is a similarity with potassium in that within families iron increases more or less linearly with increasing weight, and the joint regression is significant $(\mathrm{P}=<\mathrm{0} \cdot \mathrm{OI})$. In the case of iron, however, the regression slopes of families unlike those for potassium are very different from one another $(\mathrm{P}=<0.01)$. In $P 6 b=0.00020$, in $P_{I 7} 0.00015$ and in the $F_{2}$ $0 \cdot 00006$.

In view of this inconsistency between families an analysis of covariance is clearly inappropriate for the data as a whole. The graph in fig. 3 does, however, suggest how the data can be partitioned for separate comparisons. It will be seen that for $P 6$ and $\left.P_{I}\right\rangle$ the regression slopes are very similar and, by analysis of variance, not significantly heterogeneous. On the strength of this a covariance analysis was therefore applied to the data for the two lines separately (table 4).

TABLE 4

The co-variance analysis of iron content $(\mathrm{y})$ on seedling weight $(\mathrm{x})$ in $\mathrm{P} 6$ and $\mathrm{P}_{17}$

\begin{tabular}{|c|c|c|c|c|c|c|c|c|c|c|}
\hline & & SSy & $\mathrm{S} x y$ & $\mathrm{SS} y$ & SS regr. & SS res. & & & & \\
\hline Total . & . & $16,923 \cdot 08$ & $1 \cdot 4155$ & 0.000479 & 0.000118 & $0.00036 \mathrm{I}$ & $\mathbf{N}$ & MS & VR & $\mathbf{P}$ \\
\hline Genotypes & - & $5,927 \cdot 88$ & -0.3500 & 0.000009 & $\ldots$ & 0.000174 & I & 0.000174 & $24 \cdot 86$ & 0.001 \\
\hline Error . & . & $10,995 \cdot 20$ & $1 \cdot 7655$ & 0.000470 & 0.000283 & 0.000187 & 25 & 0.000007 & $\ldots$ & $\ldots$ \\
\hline
\end{tabular}

From it we may conclude, as for the other minerals, that there is significant variation in iron content between genotypes of corresponding weights $(P=<0.001)$.

A second comparison, between $P_{I 7}$ and the $F_{2}$, reveals a different sort of variation. Both families represent a similar range of seedling weights and their average iron contents are identical. The regression slopes, however, are significantly different $(P=0 \cdot 02-0 \cdot 01)$. It follows that not only does iron content vary independently of weight but also the rate at which it varies is apparently determined by the genotype.

To summarise, the results for iron confirm that the genotype determines variation in mineral composition of seedlings at comparable weights. They go further in suggesting that the rate of increase of mineral content which is to be expected with increasing weight is also determined genetically and may vary between genotypes.

\section{DISCUSSION}

The present evidence reveals heritable variation in the mineral composition of plants derived from the same initial population. There is every reason to suppose, therefore, that the nature of this variation 
in populations will depend upon and will respond to the influence of selection.

What this variation signifies in physiological or developmental terms is not clear. One may suggest three main, not necessarily exclusive, possibilities.

I. The requirement for a particular mineral during a given increase in plant weight is greater in some genotypes than others, as Lyness has established in maize (1936).

2. The mineral requirement is the same but the mineral may be accumulated, unused, to a greater degree in different genotypes, as a result, for example, of differences in rates of mineral absorption (see Rabideau, Whaley and Heimsch, 1950).

3. Developmental differences from the morphological standpoint determine the differences in mineral composition, particularly where the balance of organs and tissues, which themselves have characteristic mineral compositions, are highly dissimilar. It is worth noting in this connection that although the rye seedlings investigated represent outwardly the same, three tiller, stage of differentiation and development the classification is, at best, a crude one, and even if we assume an identical morphology for the seedlings in all three families, at a cellular level their varying mineral composition may well be related to, and even determine, morphological divergence at a later stage of development.

Such possibilities need to be, and can be, tested. For the present, however, one can but reaffirm the heritability of plant mineral composition and emphasise that even within a population there are possibilities for change by selection, possibilities that no doubt play a prominent part in the adaptation of natural populations and which may, perhaps, play a greater part in plant breeding practice.

\section{SUMMARY}

I. Comparisons of potassium, phosphorus and iron content were made between seedlings of three rye families, two inbred lines and an $F_{2}$, derived from the same commercial variety.

2. In all families the total mineral content of the seedlings increases with weight. For potassium and iron the increase is linear, for phosphorus, curvilinear.

3. There is evidence that the rate of change of iron content with weight is different in different families.

4. For all three minerals the content varies between different genotypes of corresponding weights. The significance of such heritable variation is briefly discussed.

Acknowledgment.-We are greatly indebted to Dr Alan Durrant for his help with the statistics. 


\section{REFERENCES}

JaCkson, M. L. 1958. Soil Chemical Analysis. Constable, London.

LYNESS, A. S. 1936. Varietal differences in the phosphorus feeding capacity of plants. Plant Physiol., II, 665-688.

RABIDEAU, G. S., WHALEY, W. G., AND HEIMSCH, c. 1950. The absorption and distribution of radioactive phosphorus in two maize inbreds and their hybrid. Amer. 7. Bot., 37, 93-99.

SMITH, G. S., MCCURDY, W. H., AND DIEHL, H. 1952. The colorimetric determination of iron in raw and treated municipal water supplies by use of $4: 7$-diphenylI : ro-phenanthroline. The Analyst, 77, 418-422.

WAGNer, R. P., AND MtTcheli, H. x. 1955. Genetics and Metabolism. Wiley, New York. 